\title{
XIII. On the temperature of a room indicatedly two thermometers at different altitudes
}

\author{
John Murray F.L.S. M.W.S.
}

To cite this article: John Murray F.L.S. M.W.S. (1822) XIII. On the temperature of a room indicatedly two thermometers at different altitudes, Philosophical Magazine Series 1, 59:285, 51-53, DOI: $10.1080 / 14786442208652677$

To link to this article: http://dx.doi.org/10.1080/14786442208652677

里 Published online: 27 Jul 2009.

Submit your article to this journal $₫$

Џ Article views: 2

Q View related articles ๘ 


\title{
[ $5 \mathbf{l l}]$
}

XIII. On the Temperative of a Room indicated ly two Thermometers at different Allitudes. By John MUrray, F.L.S. M.W.S. \&ैc. Eัc.

\author{
To Dr. Tilloch.
}

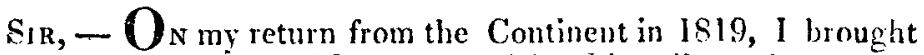
with me Breguet's " Thtrmomètre Mélaliqune," - -an instrument susceptible of the most delicate sensibility, and with which I have made many interesting experiments. In a still room without a fire, in the summer months, it readily communicated the differcnce in temperature between the floor and a chair, and this last and the table.

The phæulomena indueed me to make a series of experiments on the difference of temperature indicated by two thermometers at different altitudes, yet otherwise under similar circumstances.

In the first serics of experiments made at Nottingham from the 21 st to the 28 th October last inclusive, I was surprised to find, that any deviation from its uniformity had an innediate relation to the radiation of the terrestrial temperature to the heavens;indeed, I am much deceived if the difference in question may not be found as accurate a guide as the barometer itself. Since I came to Loudon, I have kept a pretty accurate register of the difference between two thermometers : one placed with its bulb on the floor, and the other suspended $6 \frac{1}{2}$ feet above it, embracing the period betweeen $5 \mathrm{th}$ and $24 \mathrm{th}$ November. Ou the 11th I began first to note the phronomena of the weather in correspondence with these changes, and it will be there seen, that when the difference exceeds 20 to $2 \cdot 5 \mathrm{~F}$. the weather has been variable and wet. The following comprise the results of the observations in a tabular form.

The following experiments were made at Nottingham with thin calico curtains to the windows of my room.

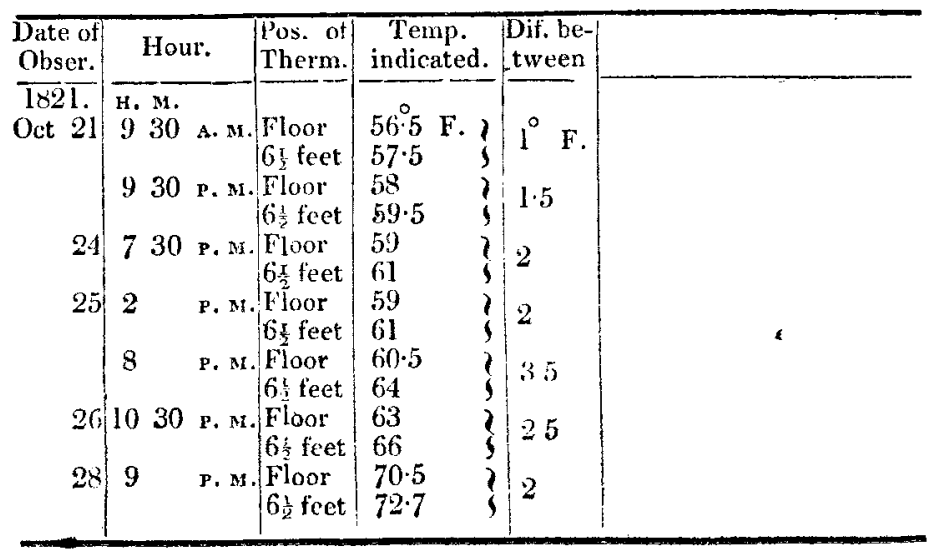


Note of experiments made in London, with two Thermometers at different altitudes.-Shutters of wood to the room.

\begin{tabular}{|c|c|c|c|c|c|c|}
\hline \multirow{5}{*}{$\begin{array}{l}\text { Date of } \\
\text { Obser. } \\
1821 . \\
\text { Nov. } 5\end{array}$} & \multirow{3}{*}{$\begin{array}{l}\text { Period of } \\
\text { Day. } \\
\frac{\text { H. M. }}{930 \text { A. }}\end{array}$} & \multirow{3}{*}{$\mid \begin{array}{l}\text { Pos. of } \\
\text { Therm. } \\
\text { Floor } \\
6 \text { I feet }\end{array}$} & \multicolumn{2}{|c|}{$\begin{array}{l}\text { Temp. } \\
\text { indicated. }\end{array}$} & \multirow{3}{*}{$\begin{array}{l}\text { Dif. be- } \\
\text { tween. } \\
0 \\
0.5 \mathrm{~F}\end{array}$} & Weather. \\
\hline & & & & & & \\
\hline & & & $\begin{array}{l}\mathbf{5 3 . 5} \text { F } \\
55\end{array}$ & & & \\
\hline & 7 р. M. & Floor & $55 \cdot 5$ & & $3 \cdot 5$ & \\
\hline & 9015 & $\begin{array}{l}8 \frac{1}{2} \text { feet } \\
\text { Floor }\end{array}$ & $\begin{array}{l}59 \\
53\end{array}$ & 3 & & \\
\hline 3 & ( & $6 \frac{\mathrm{I}}{2}$ feet & 55.25 & & 2.26 & \\
\hline & 930 P. $\mathrm{M}$ & Floor & $54 \cdot 5$ & 1 & $2 \cdot 5$ & \\
\hline 7 & A. $M$. & $\begin{array}{l}0, t e e t \\
\text { Floor }\end{array}$ & $51 \cdot 5$ & 3 & 0.5 & \\
\hline & & $6 \frac{x}{2}$ feet & 57 & j) & 20 & \\
\hline & 5 Р. м. & $\begin{array}{l}\text { Floor } \\
6 \pm \text { feet }\end{array}$ & $\begin{array}{l}54 \\
56 \cdot 5\end{array}$ & $\eta$ & $2 \cdot 5$ & \\
\hline 8 & 930 А. M. & Floor & $\begin{array}{l}55 \cdot 5 \\
58\end{array}$ & 3 & $2 \cdot 5$ & \\
\hline & $615 \mathrm{P.M}$ & Floor & $\begin{array}{l}55.5 \\
59\end{array}$ & 3. & $3 \cdot 5$ & \\
\hline 9 & 930 A. M. & $\mid \begin{array}{l}\text { nateet } \\
\text { Floor }\end{array}$ & $\begin{array}{l}29 \\
54\end{array}$ & 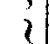 & 2.5 & \\
\hline & & $6 \frac{x}{2}$ feet & $56^{\circ} 5$ & 5 & & \\
\hline & 530 Р. $\mathrm{Mr}$ & $\begin{array}{l}\text { Floor } \\
6 x \text { feet }\end{array}$ & $\begin{array}{l}55 \\
57\end{array}$ & 9 & 2 & \\
\hline 10) & $1030 \mathrm{~A} . \mathrm{N}$ & Floor & $\begin{array}{l}56 \\
58.5\end{array}$ & $?$ & $2 \cdot 5$ & \\
\hline & 730 Р. $\mathrm{M}$ & $\mid \begin{array}{l}\text { Floor } \\
6 \text { f feet }\end{array}$ & $\begin{array}{l}59 \cdot 5 \\
62 \cdot 5\end{array}$ & 3 & 3 & \\
\hline 11 & 930 A. $\mathrm{Mr}$. & $\begin{array}{l}\text { Floor } \\
6 \frac{\mathrm{r}}{2} \text { feet }\end{array}$ & $\begin{array}{l}59 \cdot 5 \\
60 \cdot 5\end{array}$ & $?$ & 1 & \\
\hline & 8 p. M. & $\begin{array}{l}\text { Floor } \\
6 \frac{\pi}{2} \text { feet }\end{array}$ & $\begin{array}{l}635 \\
69\end{array}$ & $\xi$ & $5 \cdot 5$ & Continued rain \\
\hline 12 & 930 A. M. & $\begin{array}{l}\text { Floor } \\
6 \mathrm{x} \text { feet }\end{array}$ & $\begin{array}{l}59 \cdot 5 \\
62\end{array}$ & 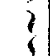 & $2 \cdot 5$ & Fine \\
\hline & 530 P. M. & $\begin{array}{l}\text { Fluor } \\
6 \mathrm{x} \text {, feet }\end{array}$ & $\begin{array}{l}59 \\
6 \mathrm{I} \cdot 5\end{array}$ & $\xi$ & $2 \cdot 5$ & Clear evening \\
\hline 13 & 915 A. M. & $\begin{array}{l}\text { Floor } \\
6 \frac{2}{2} \text { feet }\end{array}$ & $\begin{array}{l}56 \\
58\end{array}$ & $\xi$ & 2 & Foggy \\
\hline & 930 р. м. & $\begin{array}{l}\text { Floor } \\
6 \frac{\mathrm{x}}{2} \text { feet }\end{array}$ & $\begin{array}{l}61 \\
65 \cdot 5\end{array}$ & $?$ & 4 & Slight rain, and during the \\
\hline 14) & $930 \mathrm{A.} \mathrm{M.}$ & $\mid \begin{array}{l}\text { Pioor } \\
\text { bi f feet }\end{array}$ & $\begin{array}{l}62 \cdot 5 \\
65\end{array}$ & ? & $2 \cdot 5$ & Cloudy \\
\hline & 6 P. M. & $\begin{array}{l}\text { Floor } \\
6 \text { I feet }\end{array}$ & $\begin{array}{l}64 \\
67 \cdot 5\end{array}$ & $?$ & $3 \cdot 5$ & Rain \\
\hline & 10 P. M. & $\mid \begin{array}{l}\text { Floor } \\
6 \frac{1}{2} \text { feet }\end{array}$ & $\begin{array}{l}64 \\
68\end{array}$ & 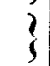 & 4 & Rain \\
\hline 15 & 10, A. M. & $\begin{array}{l}\text { Floor } \\
6, \frac{x}{2} \text { feet }\end{array}$ & $\begin{array}{l}64 \\
68\end{array}$ & $?$ & 4 & Rain \\
\hline & 630 г.м. & $\begin{array}{l}\text { Floor } \\
6 \text { f feet }\end{array}$ & $\begin{array}{l}61 \\
68\end{array}$ & 3 & 4 & Rain \\
\hline ]6: & 930 а. м. & $\begin{array}{l}\text { Floor } \\
9 \frac{1}{2} \text { feet }\end{array}$ & $\begin{array}{l}61.25 \\
61 \cdot 75\end{array}$ & 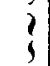 & $3 \cdot 5$ & Cloudy and rain \\
\hline & $3 \quad$ F. $\mathrm{s}$ & $\begin{array}{l}\text { Floor } \\
\text { fit fect }\end{array}$ & $\begin{array}{l}63 \\
67\end{array}$ & $?$ & 4 & Rain \\
\hline
\end{tabular}


by two Thermometers at different Alititudes.

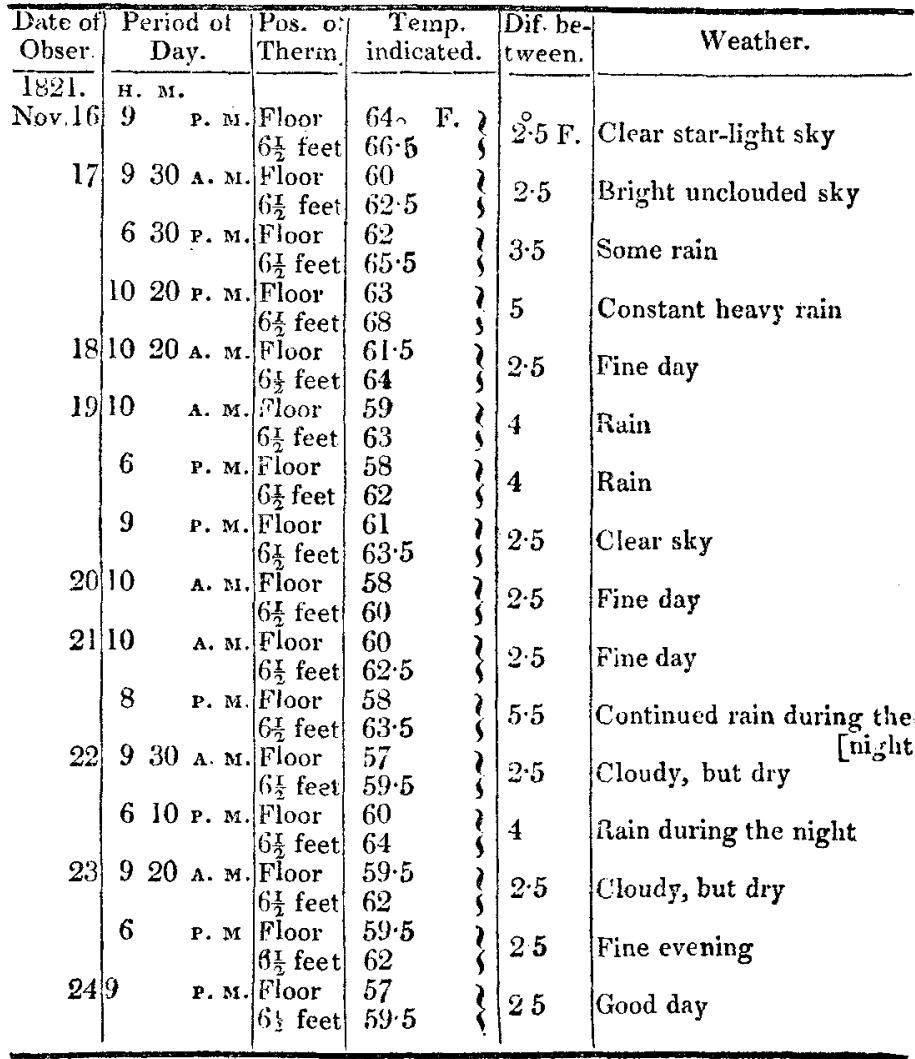

I have only to regret occasional omissions, and that my avocations did not permit me to attend to more regular intervals of time.-The question appears to me to be a curious one, and to solicit, further and more delicate attention. The correspondence is remarkable, though it will doubtless be violated by circumstances, which in the present state of our meteorological science cannot perhaps be always or altogether estimated.

I have the honour to be, sir,

Your most obedient and very humble servant,

Surry Institution, January 11, 1822.

J. Murray. 